EGU2020-3462

EGU General Assembly 2020

(c) Author(s) 2020. This work is distributed under

the Creative Commons Attribution 4.0 License.

\title{
Error assessment of precipitation products based on the elevations and extreme events
}

\author{
Ehsan Sharifi ${ }^{1}$, Wouter Dorigo ${ }^{2}$, and Josef Eitzinger ${ }^{3}$ \\ ${ }^{1}$ Hydrology Section, GFZ German Research Center for Geosciences, Potsdam, Germany (ehsan.sharifi@gfz-potsdam.de) \\ ${ }^{2}$ Department of Geodesy and Geo-Information, Vienna University of Technology (TU-Wien), Vienna, Austria \\ (wouter.dorigo@geo.tuwien.ac.at) \\ ${ }^{3}$ Department of Meteorology and Climatology, University of Natural Resources and Life Sciences (BOKU), Vienna, Austria \\ (josef.eitzinger@boku.ac.at)
}

Accurate precipitation measurement is crucial for hydrological modeling and weather forecasting. During the last decade, considerable progress has been made in satellite-based precipitation products that could be a potential source as inputs in hydro-meteorological and agricultural models, which are essential especially over the mountains area or in basins where ground gauges are generally sparse or nonexistent. This study comprehensively examined the performance of several newly released precipitation products, i.e., MSWEP-V2.2, IMERG-V05B, IMERG-V06A, IMERGV05-RT, ERA5, and SM2RAIN-ASCAT, with emphasis on their performance based on elevation and extreme events. The analysis has been conducted at daily time-scales over Austria for the period June 2014-December 2015, using a dense network of gauges (882 stations) as a reference. Since Austria characterized by complex terrain and a big difference in altitude over the country, the annual mean precipitation range significantly varies with elevation and climate conditions. Therefore, the microclimate can be created due to rapid changes in elevation which cause obstruct the air mass movement or this rapid changes in elevation can cause the updraft of the air mass over the mountains to create orographic rainfall.

The results showed that the number of extreme days is double over the Alpine area in comparison to low altitude regions. The $90 \%$ percentile level of wet days $(P \geq 0.1 \mathrm{~mm})$ as the R90th of the stations showed the maximum values at high-elevation areas (Alpine mountains). The spatial distribution of the R90th for MSWEP, IMERG-V05B, and -V06a were rather similar to observations with higher number of days for the precipitation threshold above 90th percentile over the south part of Austria. In contrast, ERA5 underestimated the frequency of the extreme events over the big part of the south region, while showed higher number of extreme days over north Austria. Moreover, SM2RAIN, displayed underestimation of the R90th, almost over the whole country. It was evident that with the increase of elevation, the mean RMSE, MAE, and bias increase and CC decreases. With respect to heavy precipitation ( $P>10 \mathrm{~mm} /$ day), MSWEP compare to other products demonstrate advantages in detecting precipitation events with a higher spatial average of probability of detection (POD) and lower false alarm ratio (FAR) scores skill (0.74 and 0.28 ), while SM2RAIN and ERA5 reveal lower POD (0.35) and higher FAR (0.56) in this precipitation range in comparison with other products. However, according to our analysis of the considered products, 
MSWEP-V2.2, followed by IMERG-V06A and -V05B, are the most suitable for driving hydrometeorological, agricultural, and other models over mountainous terrain.

Reference: Sharifi, E., Eitzinger, J., Dorigo, W. (2019). Performance of the State-Of-The-Art Gridded Precipitation Products Over Mountainous Terrain. A Regional Study Over Austria. Remote Sensing 11(17), 2018, https://doi.org/10.3390/rs11172018. 